\title{
Development of the Wheelchair User's Shoulder Pain Index (WUSPI)
}

\author{
KA Curtis ${ }^{1}$, KE Roach ${ }^{2}$, E Brooks Applegate ${ }^{2}$, T Amar ${ }^{2}$, CS Benbow ${ }^{2}$, TD Genecco ${ }^{2}$ and $\mathrm{J}_{\text {Gualano }}^{2}$ \\ ${ }^{1}$ Department of Physical Therapy, California State University Fresno, 2345 E San Ramon Ave, Fresno, CA \\ 93740-0029, USA; ${ }^{2}$ Department of Orthopaedics and Rehabilitation, University of Miami School of Medicine, \\ Florida, USA
}

\begin{abstract}
Chronic shoulder pain is a frequently reported phenomenon in individuals who use wheelchairs as their primary means of mobility. No indices are currently available which detect difficulties in performing daily activities due to shoulder discomfort in a largely independent population of wheelchair users. The Wheelchair User's Shoulder Pain Index (WUSPI) was designed to measure shoulder pain in individuals who use wheelchairs. A pilot index was created to measure shoulder pain and related difficulty during basic and instrumental activities of daily living. The instrument was administered to 64 wheelchair users at an athletic event. Analyses of internal consistency and interitem correlations were used to revise and refine the original instrument. Individual item analysis revealed that the subjects in this study experienced the most shoulder pain when wheeling up an incline or on outdoor surfaces, when lifting an object from an overhead shelf, when trying to sleep, when transferring from tub to wheelchair and when washing their backs. The final 15 -item index shows high internal consistency. This instrument is useful for both clinical and research purposes to detect and monitor shoulder pain and accompanying loss of function by wheelchair users.
\end{abstract}

Keywords: spinal cord injury; aging; paraplegia; activities of daily living; shoulder pain; measurement; index

\section{Introduction}

Cross sectional studies have reported that $60-100 \%$ of long term wheelchair users experience shoulder pain..$^{1,2}$ This may be due to several factors. Wheelchair users rely largely on the upper extremities for mobility and transfers ${ }^{1,3,4}$ which eventually results in degenerative structural or physiological joint changes. ${ }^{5}$ Researchers have reported that wheelchair users demonstrate chronic degenerative injuries to soft tissues, including impingement syndromes, rotator cuff tears, sprains, strains and avascular necrosis, as well as evidence of radiological degenerative changes in the shoulder joint. $^{5-9}$

The independence of wheelchair users depends on the integrity of their upper limbs. ${ }^{2,4}$ Several studies have shown that shoulder pain is a limiting factor in the daily activities of individuals with paraplegia, especially during transfers, wheelchair propulsion, reaching, lifting, driving and loading the wheelchair into the car. ${ }^{2,4,5}$

Many indices are available to measure generalized pain or functional impairment in individuals with chronic disabilities. ${ }^{10-13}$ Roach et al developed an index to quantify shoulder pain and difficulty during functional activities in an ambulatory population. ${ }^{13}$ However, at the time of this study no index was available to measure shoulder pain and difficulty in wheelchair users.

Although shoulder pain may not initially limit an individual's ability to perform activities independently, it may have other functional costs to the wheelchair user, such as decreased speed or efficiency of movement, rapid fatigue or low tolerance for prolonged work or leisure activity, decreased cardiorespiratory endurance, chronic postural deviations or elimination of positions or activities that are associated with pain. Secondary complications such as obesity, scoliosis, skin breakdown and contractures are often associated with decreased activity levels in wheelchair users. It is therefore important to accurately identify shoulder pain which interferes with function in wheelchair users and to quantify its severity. This article describes the development of the Wheelchair User's Shoulder Pain Index (WUSPI), a shoulder pain index designed to measure the severity of shoulder pain associated with functional activity in individuals who use wheelchairs.

\section{Methods}

\section{Instrumentation}

Preliminary instrument development A 38-item, twopart functional index, modeled after the Shoulder Pain and Disability Index (SPADI) developed by Roach et al,${ }^{13}$ was created to assess two measurement dimensions: (1) difficulty associated with shoulder problems during functional activities and (2) shoulder pain with functional activities in individuals who use wheelchairs. The SPADI was modified by including common 
functions and activities of daily living performed by the wheelchair user, drawn from a comprehensive literature review. To establish content validity, we asked a group of long term wheelchair users to review our list of selected wheelchair activities and to suggest additional functional activities associated with chronic shoulder pain.

A $10 \mathrm{~cm}$ visual analog scale (VAS) anchored at 'no difficulty' and 'so difficult require help' was used to measure the 19 items addressing the difficulty dimension during functional activities. An identical VAS anchored at 'no pain' and 'worst pain ever experienced' was used to measure the 19 items addressing the pain dimension during functional activities. Thus, the lowest possible score for each item was zero and the highest possible score was 10 .

A demographic questionnaire was developed to identify factors relevant to the subject's lifestyle and shoulder dysfunction, including items to assess age, gender, marital status, occupational and recreational activities, nature of their primary disability, and years since the onset of their disability. Medical history data collected included the presence or absence of past or current shoulder pain, the shoulder(s) involved, treatment received, and presence of other hand and elbow dysfunction.

\section{Subjects and procedures}

A convenience sample of 64 subjects was drawn from a group of more than 400 athletes attending a large wheelchair athletic event for paralyzed veterans. To participate in the study, subjects met two criteria: they (1) used a wheelchair as their primary means of mobility and (2) used a wheelchair for at least 1 year prior to their participation.

All subjects who met the inclusion criteria and agreed to participate then signed a consent form and were given instructions for completion of the instrument. Experimenters were available to answer any questions the subjects had regarding the wording on the index. Subjects took approximately $10 \mathrm{~min}$ to complete the index.

\section{Data analysis}

The subject's score on each item was calculated by measuring their response on the VAS in $\mathrm{cm}$. Index scores were calculated for each of the pain and difficulty dimensions and for the total index by adding the component individual item scores. Pearson's correlations were performed between items and total scores in the difficulty and pain dimensions. Internal consistency was estimated by calculation of Cronbach's alpha for each of the 19-item pain and difficulty dimensions separately and for the 38-item instrument as a whole.

\section{Results}

Subjects

The average age of the 64 subjects was 42.9 years ( \pm 11.0 years) ranging from 23 to 68 years of age. The subjects included 62 men and two women. Twenty one $(33 \%)$ subjects were employed, $10(16 \%)$ were students, four $(6 \%)$ worked as volunteers and $29(45 \%)$ were unemployed or retired. The majority $(89 \%)$ of subjects reported spinal cord injury (SCI) as their primary disability, with the remainder of the subjects reporting poliomyelitis, amputation, cerebrovascular accidents, and multiple sclerosis. Of the 57 subjects with SCI, 13 had cervical lesions, 38 had thoracic lesions, and six had lumbar lesions. Subjects averaged 14.3 years of wheelchair use ( \pm 8.5 years) with a range from 1.5 to 45 years.

\section{Shoulder pain}

Forty seven (73\%) of the 64 subjects reported having current shoulder pain. Of these 47 subjects with current pain, $12(25.5 \%)$ had pain in the left shoulder, 12 $(25.5 \%)$ had pain in the right shoulder and $23(49.0 \%)$ had pain bilaterally. Only $17(27 \%)$ subjects reported experiencing no current shoulder pain.

These subjects reported most intense pain when performing functional activities requiring extremes of shoulder range of motion, overhead positioning or a high level of upper extremity strength. The most painful activities included wheeling up inclines, reaching to an overhead shelf, performing non-level transfers and washing one's back.

\section{Internal consistency and interitem correlations}

Internal consistency of the 38-item pilot questionnaire was calculated using Cronbach's alpha. The index demonstrated high internal consistency $(\alpha=0.97)$, as did each of the difficulty ( $\alpha=0.96)$ and pain $(\alpha=0.97)$ dimensions. We compared subject ratings of pain and difficulty for each activity and found many high interitem correlations between the pain and difficulty dimensions of the scale (Table 1).

\section{Index revisions}

Elimination of the difficulty dimension Ease of administration of any index is a desirable feature. Our aim was to create an easily self-administered measurement tool. Given the potential of limited hand function in this population and limitations of personnel time for instrument administration and scoring, we sought to shorten and simplify the index further.

In wheelchair users, it may be hard to differentiate between difficulty due to absent or impaired neurological function and difficulty due to shoulder problems. The measurement of shoulder pain during functional activities may better reflect the influence of a shoulder problem than does the measurement of difficulty. Since there were high interitem correlations and high internal consistency of each dimension we decided to eliminate the 19 item difficulty dimension of the index. The elimination of the difficulty dimension left 19 items intended to measure the subject's level of shoulder pain when performing functional activities.

We also compared pain scores on items that involved similar motions or abilities. Four items with high 
Table 1 Pilot instrument item scores and interdimensional correlations ( $n=64$ subjects)

\begin{tabular}{|c|c|c|c|c|c|c|}
\hline \multirow[t]{3}{*}{ Items } & \multicolumn{6}{|c|}{ Measurement dimensions } \\
\hline & \multicolumn{2}{|c|}{ Difficulty } & \multicolumn{2}{|c|}{ Pain } & \multicolumn{2}{|c|}{ Correlation } \\
\hline & Mean & $S D$ & Mean & $S D$ & $r$ & $P$ \\
\hline \multicolumn{7}{|l|}{ Transfers } \\
\hline Bed-wheelchair & 1.15 & 2.07 & 1.56 & 1.98 & 0.743 & 0.0001 \\
\hline Car-wheelchair & 1.87 & 2.31 & 2.03 & 2.15 & 0.712 & 0.0001 \\
\hline Tub/shower-wheelchair & 1.88 & 2.43 & 2.11 & 2.19 & 0.686 & 0.0001 \\
\hline Load wheelchair in car & 1.80 & 2.28 & 2.06 & 2.2 & 0.791 & 0.0001 \\
\hline \multicolumn{7}{|l|}{ Wheelchair mobility } \\
\hline Indoor propulsion & 1.13 & 1.69 & 1.58 & 1.92 & 0.696 & 0.0001 \\
\hline$>10$ min duration & 1.23 & 1.82 & 1.59 & 2.06 & 0.691 & 0.0001 \\
\hline Up ramp/incline & 1.93 & 2.28 & 2.30 & 2.48 & 0.839 & 0.0001 \\
\hline Uneven surfaces (curb/grass) & 2.17 & 2.38 & 2.48 & 2.60 & 0.825 & 0.0001 \\
\hline \multicolumn{7}{|l|}{ Self-care } \\
\hline Lift object from overhead & 2.48 & 2.65 & 2.32 & 2.55 & 0.584 & 0.0001 \\
\hline Bowel/bladder care & 1.44 & 2.37 & 1.13 & 1.85 & 0.642 & 0.0001 \\
\hline Put on pants & 1.37 & 1.99 & 1.19 & 1.80 & 0.633 & 0.0001 \\
\hline Put on T-shirt & 1.25 & 1.77 & 1.51 & 1.96 & 0.716 & 0.0001 \\
\hline Put on button-down shirt & 1.22 & 1.91 & 1.24 & 1.72 & 0.651 & 0.0001 \\
\hline Wash hair/head & 1.02 & 1.82 & 1.33 & 1.97 & 0.851 & 0.0001 \\
\hline Wash back & 2.76 & 2.91 & 2.09 & 2.57 & 0.648 & 0.0001 \\
\hline \multicolumn{7}{|l|}{ General activities } \\
\hline Work/school activities & 1.47 & 1.90 & 1.64 & 1.96 & 0.717 & 0.0001 \\
\hline Driving & 0.99 & 1.57 & 1.40 & 1.82 & 0.731 & 0.0001 \\
\hline Household chores & 1.58 & 1.89 & 1.60 & 1.94 & 0.603 & 0.0001 \\
\hline Sleeping & 2.02 & 2.32 & 2.31 & 2.64 & 0.891 & 0.0001 \\
\hline Total scores & 33.94 & 28.65 & 36.12 & 31.50 & 0.842 & 0.0001 \\
\hline Cronbach's alpha & 00.96 & & 00.98 & & & 0.0001 \\
\hline
\end{tabular}

correlation to items measuring similar motions (with $r>0.85$ ) were eliminated, leaving the final 15 -item functional index to measure shoulder pain during functional activities in individuals who use wheelchairs (Table 2).

Reanalysis of internal consistency of the 15-item final WUSPI showed that Cronbach's alpha was unchanged following these revisions $(\alpha=0.97)$.

\section{Discussion}

\section{WUSPI index revisions}

The statistical analyses of the preliminary 38-item instrument by Pearson's correlation and Cronbach's alpha indicated a highly intercorrelated instrument with high internal consistency in both of the two measurement dimensions of difficulty and pain for 19 functional activities of wheelchair users. Unlike studies on able bodied subjects, ${ }^{15}$ the pain and difficulty items in this index did not seem to represent separate constructs. This apparent unidimensionality and the high interitem and interdimension correlations led to our decision to revise and shorten the instrument considerably. Repeated analyses of internal consistency show that the final 15-item WUSPI is a simple, cohesive instrument which reflects the functional costs of shoulder pain in wheelchair users.
The WUSPI is easily administered as a paper and pencil measure, which can be completed in person or by mail. Completion takes only $5 \mathrm{~min}$. Because of the instrument's simplicity, it is easily translated into other languages and the visual analog scale eliminates the need to label an ordinal scale with multiple descriptors.

The WUSPI is a valuable tool that can be used to detect and monitor upper extremity musculoskeletal complications in wheelchair users. It provides unique information, in that it measures pain across a variety of functional activities which are specific to wheelchair users.

Additionally, the WUSPI should be more sensitive than functional measures which use criteria of independence and dependence. The WUSPI differs from functional scales in that it measures subjective change in individuals with chronic disabilities who are functioning independently, but with difficulty, due to problems such as pain or limitation of range of motion. This instrument is able to detect even small levels of shoulder pain, in a way that is practical and relevant to this population and their health care consultants.

\section{Conclusion}

The Wheelchair Users Shoulder Pain Index (WUSPI) was developed to measure shoulder pain causing 
Table 2 Correlation of four eliminated items to final items

\begin{tabular}{|c|c|c|c|c|}
\hline \multirow[t]{2}{*}{ Final items } & \multicolumn{4}{|c|}{ Four eliminated items } \\
\hline & $\begin{array}{c}\text { Indoor wheelchair } \\
\text { propulsion } \\
r\end{array}$ & $\begin{array}{c}\text { Wheelchair propulsion } \\
\text { uneven surfaces } \\
r\end{array}$ & $\begin{array}{c}\text { Perform bowel/bladder } \\
\text { care } \\
r\end{array}$ & $\begin{array}{c}\text { Wash hair/head } \\
r\end{array}$ \\
\hline \multicolumn{5}{|l|}{ Transfers } \\
\hline Bed-wheelchair & 0.785 & 0.690 & 0.748 & 0.765 \\
\hline Car-wheelchair & 0.690 & 0.754 & 0.592 & 0.716 \\
\hline Tub/shower-wheelchair & 0.761 & 0.768 & 0.649 & 0.804 \\
\hline Load wheelchair into car & 0.671 & 0.759 & 0.733 & 0.797 \\
\hline \multicolumn{5}{|l|}{ Wheelchair mobility } \\
\hline$>10$ min duration & 0.900 & 0.802 & 0.701 & 0.718 \\
\hline Up ramp/incline & 0.869 & 0.946 & 0.621 & 0.599 \\
\hline \multicolumn{5}{|l|}{ Self-care } \\
\hline Lift objects from overhead & 0.737 & 0.792 & 0.707 & 0.778 \\
\hline Put on pants & 0.630 & 0.520 & 0.877 & 0.783 \\
\hline Put on T-shirt & 0.750 & 0.725 & 0.808 & 0.854 \\
\hline Put on button down shirt & 0.700 & 0.636 & 0.617 & 0.744 \\
\hline Wash back & 0.725 & 0.789 & 0.629 & 0.768 \\
\hline \multicolumn{5}{|l|}{ General activities } \\
\hline Work/school activities & 0.675 & 0.752 & 0.662 & 0.835 \\
\hline Driving & 0.537 & 0.532 & 0.661 & 0.650 \\
\hline Household chores & 0.680 & 0.715 & 0.639 & 0.765 \\
\hline Sleeping & 0.641 & 0.655 & 0.506 & 0.589 \\
\hline
\end{tabular}

limitation of function in individuals who use wheelchairs. This index can be used clinically for evaluation and treatment by assessing shoulder pain during function in wheelchair users. The instrument is also appropriate for use as an outcome measure in research to monitor the effects of various interventions to prevent chronic shoulder pain and loss of function in the wheelchair using population, such as wheelchair modifications or shoulder strengthening programs.

\section{Acknowledgements}

This study was funded in part by the Florida Paralyzed Veterans of America. The authors also acknowledge the support of the Paralyzed Veterans of America (PVA) for permitting us to conduct this study at the 13th National Veterans Wheelchair Games in San Antonio, TX. The authors would also like to express their appreciation to Rob Voorhees and Amparo Rally for their assistance with manuscript preparation.

\section{References}

1 Nichols P, Norman PA, Ennis JR. Wheelchair user's shoulder. Scand J Rehabil Med 1979; 11: 29-32.

2 Pentland WE, Twomey LT. The weight bearing upper extrem- ity in women with long term paraplegia. Paraplegia 1991; 29: 521-530.

3 Waring WP, Maynard FM. Shoulder pain in acute traumatic quadriplegia. Paraplegia 1991; 29: 37-42.

4 Gellman H, Sie I, Waters RL. Late complications of the weight bearing upper extremity in the paraplegic patient. Clin Orthop 1988; 223: 132-135.

5 Bayley JC, Cochran TP, Sledge CB. The weight-bearing shoulder: The impingement syndrome in paraplegics. $J$ Bone Joint Surg Am 1987; 69: 676-678.

6 Wylie EJ, Chakera TM. Degenerative joint abnormalities in patients with paraplegia of duration greater than 20 years. Paraplegia 1988; 26: 101-106.

7 Curtis KA, Dillon DA. Survey of wheelchair athletic injuries: Common patterns and prevention. Paraplegia 1985; 23: $170-175$.

8 Burnham RS et al. Shoulder pain in wheelchair athletes; the role of muscle imbalance. Am J Sports Med 1993; 21: 238-242.

9 Nilsen R, Nygaard P, Bjerholt PG. Complications that may occur in those with spinal cord injuries who participate in sport. Paraplegia 1985; 23: 152-158.

10 Melzack R. The McGill Pain Questionnaire: Major properties and scoring methods. Pain 1975; 1: 277-299.

11 Williams RC. Toward a set of reliable and valid measures for chronic pain assessment and outcome research. Pain 1988; 35: 239-251.

12 Feinstein AR. Clinimetric perspectives. J Chron Dis 1987; 40: 635-640.

13 Roach KE, Budiman-Mak E, Songsiridej N, Lertratanakul Y. Development of a shoulder pain and disability index. Arthritis Care Res 1991; 4: 143-149. 